\title{
Replacing sedentary time with physical activity or sleep: effects on cancer-related cognitive impairment in breast cancer survivors
}

Diane K. Ehlers ${ }^{1 *}$ (D) Jason Fanning ${ }^{2}$, Elizabeth A. Salerno ${ }^{3}$, Susan Aguiñaga ${ }^{4}$, Josh Cosman ${ }^{5}$, Joan Severson ${ }^{6}$, Arthur F. Kramer ${ }^{4,7}$ and Edward McAuley ${ }^{4}$

\begin{abstract}
Background: Evidence suggests reallocating daily sedentary time to physical activity or sleep confers important health benefits in cancer survivors. Despite emerging research suggesting physical activity as a treatment for cancer-related cognitive impairment $(C R C I)$, little is known about the interactive effects of behaviors across the 24-h period. The present purpose was to examine the cognitive effects of reallocating sedentary time to light-intensity physical activity, moderate-to-vigorous physical activity (MVPA), or sleep in breast cancer survivors.

Methods: Breast cancer survivors ( $N=271$, Mage $=57.81 \pm 9.50$ years) completed iPad-based questionnaires and cognitive tasks assessing demographics, health history, executive function, and processing speed (Task-Switch, Trail Making). Participants wore an accelerometer for seven consecutive days to measure their sedentary, physical activity, and sleep behaviors. Single effects (each behavior individually) and partition (controlling for other behaviors) models were used to examine associations among behaviors and cognitive performance. Isotemporal substitution models were used to test the cognitive effects of substituting 30 min of sedentary time with 30 min of light-intensity activity, MVPA, and sleep.
\end{abstract}

Results: MVPA was associated with faster Task-switch reaction time in the partition models (stay: $B=-35.31, p=0.02$; switch: $B=-48.24, p=0.004$ ). Replacing 30 min of sedentary time with 30 min of MVPA yielded faster reaction times on Task-Switch stay $(B=-29.37, p=0.04)$ and switch $(B=-39.49, p=0.02)$ trials. In Trails $A$ single effects models, sedentary behavior was associated with faster completion $(B=-0.97, p=0.03)$ and light-intensity activity with slower completion ( $B=1.25, p=0.006$ ). No single effects were observed relative to Trails $B$ completion (all $p>0.05$ ). Only the effect of MVPA was significant in the partition models (Trails $A: B=-3.55, p=0.03$; Trails $B: B=-4.46, p=0.049$ ). Replacing sedentary time with light-intensity activity was associated with slower Trails $A(B=1.55 p=0.002)$ and Trails $B(B=1.69, p=0.02)$ completion. Replacing light activity with MVPA yielded faster Trails $A(B=-4.35, p=0.02)$ and Trails $B(B=-5.23, p=0.03)$ completion.

Conclusions: Findings support previous research suggesting MVPA may be needed to improve cognitive function in breast cancer survivors. Trails findings underscore the need to dissect sedentary contexts to better understand the impact of daily behavioral patterns on CRCl. Additional research investigating the cognitive impacts of behaviors across the 24-h period is warranted.

Trial registration: This study is registered with United States ClinicalTrials.gov (NCT02523677; 8/14/2015).

\footnotetext{
* Correspondence: ehlersd@mailbox.sc.edu

'University of South Carolina, Columbia, SC 29208, USA

Full list of author information is available at the end of the article
}

(c) The Author(s). 2018 Open Access This article is distributed under the terms of the Creative Commons Attribution 4.0 International License (http://creativecommons.org/licenses/by/4.0/), which permits unrestricted use, distribution, and reproduction in any medium, provided you give appropriate credit to the original author(s) and the source, provide a link to the Creative Commons license, and indicate if changes were made. The Creative Commons Public Domain Dedication waiver (http://creativecommons.org/publicdomain/zero/1.0/) applies to the data made available in this article, unless otherwise stated. 


\section{Background}

Improvements in cancer detection and treatment have resulted in a burgeoning population of cancer survivors in the United States (US). While these improvements represent an important advancement in cancer care, researchers and clinicians face new health challenges associated with cancer survivorship and aging. Breast cancer survivors (BCS) comprise one of the largest survivor populations, with over 3 million living in the US today [1]. Unfortunately, BCS report a number of physical, emotional, and cognitive sequelae related to their cancer diagnosis and treatment. Cognitive deficits due to cancer have increasingly been recognized as a clinical research priority, with some studies suggesting up to $83 \%$ of BCS report cognitive impairment after diagnosis [2]. These impairments can be intense, disruptive, and last for durations up to 20 years after treatment ends [3]. The increasing prevalence of cancer-related cognitive impairment (CRCI), as a result of the rapidly growing population of adults at the intersection of cancer-related and age-related cognitive decline, indicate a critical need to investigate potential treatments for CRCI [4, 5].

While a number of treatment modalities have been identified [6], recent studies provide compelling evidence in support of physical activity for mitigating cognitive impairments in cancer survivors [7-9]. Ehlers and colleagues [10] found that more daily minutes of objectively measured moderate-to-vigorous physical activity (MVPA) were associated with better performance across seven tasks of executive function and working memory in a sample of 299 BCS. Marinac and colleagues [11] observed similar relationships between MVPA and processing speed in a sample of 136 postmenopausal BCS. Experimental studies provide further support of these observational findings. Zimmer and colleagues (2016), in the only review of these relationships, found that exercise training may be a promising behavioral modality for CRCI; yet, evidence is limited due to few studies in human models and poor study quality. Hartman and colleagues (2018) recently observed improvements in processing speed among BCS enrolled in a 12-week physical activity intervention compared with controls. Unfortunately, BCS spend significantly more time sedentary and less time engaged in physical activity when compared with women not diagnosed with cancer [12-14].

An emerging literature has specifically focused on the deleterious health effects of extended periods of sedentary behavior in the general adult population and cancer survivors [12, 15, 16]. Voss and colleagues [17] argued that even adequate amounts of daily MVPA may not offset the negative impacts of prolonged sitting on brain health and cognitive function. In other words, individuals who meet the federal guidelines for physical activity ( $\geq 150 \mathrm{~min}$ per week of MVPA) [18], but also engage in long bouts of sitting may still be subject to significant health risks. Additionally, a number of studies suggest sleep deprivation may be associated with accelerated cognitive decline across the lifespan [19]. Empirical studies have suggested that reallocating daily sedentary time to MVPA, light activity, or sleep may confer important benefits to physical health, well-being, and cognition in older adults [20-22]. For example, Fanning and colleagues [22], using a statistical estimation technique called isotemporal substitution modeling, observed hypothetical benefits to older adults' executive function when substituting $30 \mathrm{~min}$ of sedentary time with $30 \mathrm{~min}$ of MVPA or sleep. As the biological pathway of cancer-related cognitive decline is thought to represent an accelerated and intensified version of age-related cognitive decline, this evidence from the aging literature may be applicable to cancer survivors.

A small number of studies have explored relationships between sedentary time reallocation and health in cancer survivors. However, this research has restricted its focus to health-related quality of life (HRQoL) outcomes and findings have been mixed. For example, Phillips and colleagues [23] and van Roekel and colleagues [24] observed benefits of light-intensity physical activity and MVPA on fatigue and HRQoL in BCS and colorectal cancer survivors, respectively. Trinh and colleagues [25] also found that sedentary behavior in BCS engaging in low amounts of MVPA was associated with higher levels of fatigue, pain, and depression. Similarly, Vallance and colleagues [26], using isotemporal substitution modeling in non-Hodgkin lymphoma survivors observed significant improvements in fatigue and clinically important improvements in HRQoL when substituting sedentary activity with MVPA. As HRQoL outcomes, such as fatigue, are thought to be associated with CRCI [10, 27], studies investigating the effects of sedentary time reallocation on CRCI are warranted.

The pool of time during which one can engage in these behaviors is finite; therefore, engagement in one behavior replaces time spent in another behavior. While MVPA undoubtedly has the greatest health benefits, more research investigating interactive effects of behaviors across the 24-h period (sleep, sedentary time, light-intensity activity, MVPA) on cognitive function in cancer survivors is warranted. Surveillance data suggest BCS may participate in as little $3.7 \mathrm{~min}$ of MVPA per day, with MVPA comprising only $1 \%$ of BCS's daily wake time [12]. As such, exercise prescriptions promoting MVPA may not be the most attractive or accessible to cancer survivors compared to prescriptions promoting lower intensity physical activities [28-30]. Understanding the health benefits of behaviors across the 24-h day may improve the delivery and effectiveness of cancer rehabilitation and ultimately have greater public health impact. 
Using isotemporal substitution modeling, the purpose of the present study was to examine the estimated cognitive effects of substituting daily sedentary time with light-intensity physical activity, MVPA, or sleep. We hypothesized that reallocating $30 \mathrm{~min}$ of sedentary time per day to $30 \mathrm{~min}$ of light-intensity physical activity, MVPA, or sleep would be associated with improved performance on cognitive tasks of speed of processing and executive function.

\section{Methods}

\section{Participants and procedures}

Women aged 21 years and older who had completed treatment for breast cancer and had access to an iPad with $\mathrm{iOS} 6.1$ or later were eligible to participant in the study. Participants were recruited via the Army of Women $\odot$, BreastCancerTrials.org, social media, emailed flyers, and word of mouth. The data presented herein represent cross-sectional findings from a subsample of breast cancer survivors enrolled in a larger prospective observational study examining relationships between physical activity and cognitive function $(N=300$ of 430$)$. Specifically, interested individuals were asked to download a free iPad application (app; Digital Artefacts, Iowa City, IA) [31] designed for this study and including a series of questionnaires and cognitive tasks. All participants provided institutional review board-approved electronic informed consent prior to their participation and were instructed to complete the assessments within 14 days of signing the consent form. The entire questionnaire battery and cognitive battery (including two practice trials) were designed to be completed in approximately $45 \mathrm{~min}$ each. Participants were not required to complete all assessments within one sitting, but could complete part of the questionnaire battery or only one cognitive task at each session [10]. All participants were invited to wear the accelerometer; however, this portion of the study was optional. Therefore, the present study included only those women who agreed to wear the accelerometer and had valid physical activity data $(N=$ 300). BCS who wore the accelerometer did not differ from those who did not wear the monitor on any demographic or clinical characteristics with the exception of receipt of radiation therapy. Women who wore the accelerometer were more likely to have received radiation.

\section{Measures}

\section{Demographic and clinical information}

The questionnaire battery included measures asking participants to report their demographic information and breast cancer history. Variables included age, race/ethnicity, marital status, employment status, education, income level, and previous use of cognitive training tools; breast cancer diagnosis date, stage, estrogen receptor, and menopausal status; chemotherapy, radiation therapy, surgery, and adjuvant hormonal therapy history. These questionnaires have been used in our previous research in BCS (Additional file 1) [10].

\section{Daily activity and sleep}

Participants were mailed an accelerometer (Actigraph GT3X, Pensacola, FL) within approximately 2 months of signing the consent form (mean $=33.6 \pm 14.7$ days; Range 0-83 days). Any mailing delays were due to accelerometer availability, participant response to invitation, participant travel schedule, or device re-wear. Devices were initialized to capture movement in 1-s epochs. Participants were instructed to wear the device on a waistband on their non-dominant hip during their wake period, move it to their non-dominant wrist using a wrist band immediately before going to bed, and return it to their waist upon wakening. Women who were diagnosed with lymphedema or experienced any discomfort on their non-dominant side were asked to wear it on their dominant wrist during sleep if possible.

Each participant kept a log of their wake and bed times, which were used to filter the data for separate wear time validation and scoring between wake and sleep periods in Actilife Version 6 (Actigraph, Pensacola, FL). After filtering out sleep windows, daytime non-wear periods were defined as the presence of $\geq 60$ consecutive "zero" intensity counts. Physical activity data were considered valid if the device was worn at least $10 \mathrm{~h}$ during the participant's waking hours and on at least 4 days [32], and sleep data were considered valid if the device was worn to bed as determined by participant record of use logs and manual inspection of the data. Valid activity data were scored using Freedson cutpoints [33] and are represented as average daily minutes spent in sedentary, light, and moderate-to-vigorous activity. Sleep data were scored using the Sadeh algorithm [34] and are represented as average daily minutes of sleep. Daily time spent sleeping, sedentary, in light-intensity physical activity and in MVPA, in addition to total time (i.e., sleep + sedentary + light + MVPA), were scaled to increments of $30 \mathrm{~min}$ for modeling purposes and to aid in interpretability [22, 26, 35, 36].

\section{Cognitive function}

The cognitive testing module of the mobile app was powered by BrainBaseline $\odot$ (www.brainbaseline.com; Digital Artefacts, Iowa City, IA), a commercially-available neuropsychological testing platform. BrainBaseline $\odot$ includes standard, laboratory-based cognitive tasks with high internal consistency and test-retest reliability. Lee and colleagues [31] demonstrated the utility and initial validity of BrainBaseline $\odot$ in a sample of 15,346 individuals aged 10$60+$ years, and the platform has since been used in other 
studies [10, 37]. Raw data were downloaded from BrainBaseline $\odot$ and processed in MATLAB version 8.4 (Mathworks, Inc., Natick, MA) to calculate summary scores for each task.

The Task-Switch [38] and Trail Making [39] tasks were used to measure participants' cognitive functioning (i.e., speed of processing and executive function) in the present study. Task-switch trials began with the presentation of a pink or blue square at the center of the screen, inside of which was a number (1-4 or 6-9). Numbers were presented individually for 2500 milliseconds. When the square was blue, participants were asked to report as quickly as possible whether the number was higher or lower than 5 using one hand. When the square was pink, participants were asked to report as quickly as possible whether the number was odd or even using the other hand. Participants complete 48 trials in which the task switched randomly across trials. Task performance in the present study was measured as accuracy and reaction time on the stay trials (i.e., color presented is same as previous trial) and switch trials (i.e., color presented is different from previous trial) separately. Accuracies and reaction times were recoded as missing if participants did not achieve $50 \%$ accuracy on the task [10].

During the Trail Making task, participants used their finger to draw a line between a series of numbers and/or letters in ascending order. Trails A targets were comprised of numbers only (i.e., 1, 2, 3, etc.). During Trails B trials, participants alternated between numbers and letters in ascending order (i.e., 1, A, 2, B, etc.). Participants were instructed to finish each task as quickly as possible. Task performance in the present study was measured as the time to complete each trail. Task-Switch took about 5 min to complete, and Trail Making (both Trails A and B) took about 3 min to complete with practices. Participants were required to complete the cognitive tasks in the order in which they appeared in the app, with Trail Making immediately following the Task-Switch task.

\section{Data analysis}

Linear regression modeling was used to examine relationships among the predictor variables (sedentary time, light activity, MVPA, and sleep duration) and cognitive outcomes (accuracy and reaction time on Task-Switch and overall time on Trails A and B). Visual inspection of the partial regression plots and scatter plots of the studentized residuals against the unstandardized predict values indicated linear relationships between independent (activity behaviors and sleep) and dependent variables (cognitive performance). Inspection of standardized residual histograms and P-P plots indicated the residuals were approximately normal. Independent and dependent variables were Winsorized at 3 standard deviations from the mean due to the presence of a small number of outliers. We ran three regression models for each outcome, including single effects, partition, and isotemporal substitution models $[21,26,35]$. In the single effects model, the effect of each behavioral predictor on cognitive performance was tested without the other behavioral predictors, but was adjusted for total time. In the partition model, the effects of each behavioral predictor were tested while controlling for the other behaviors. In the isotemporal substitution model, the total time variable was included as a predictor, while the variable of reallocation (i.e., sedentary time) was excluded from the model. The coefficients were interpreted as the mean effect of replacing $30 \mathrm{~min}$ of sedentary time with $30 \mathrm{~min}$ of each respective activity, while holding the other activities constant [35]. Models were adjusted for age, months of adjuvant hormonal therapy, receipt of chemotherapy, and total time the accelerometer was worn [40]. Additional adjustment for stage of diagnosis, months since diagnosis, education level, and menopausal status did not elicit meaningful changes to the results. Statistical significance was tested at an alpha of $p \leq 0.05$. Results are presented as unstandardized coefficients and 95\% confidence intervals (CIs; Tables 2, 3, 4 and 5). All data were analyzed in SPSS 24.

\section{Results}

Participants were 271 BCS aged $28-79$ years $(M$ age $=$ $57.81 \pm 9.67$; Table 1) drawn from a larger study of 430 women. From the parent study, 300 BCS who had completed primary treatment for breast cancer wore the accelerometer during their wake period and had valid physical activity data. Fourteen participants were missing sleep data due to lymphedema, wrist swelling, or discomfort, leaving 286 women eligible for analysis. Of these, 271 completed the Task-switch task and 269 completed Trails A and B. Finally, two participants had invalid task-switch data on both the stay and switch trials due to accuracies $<50 \%$, and one had invalid switch trial data only. The flow of participants through the present study is detailed in Fig. 1.

\section{Task-switch (Tables 2 and 3)}

No significant effects of any behaviors on Task-switch stay or switch accuracy were observed across all models (all $p>0.05$; no table included). In single effects models, MVPA was marginally associated with faster reaction time on stay trials $(B=-27.45, p=0.06$; Table 2$)$ and significantly associated with fast times on switch trials $(B=-38.63, p=0.02$; Table 3$)$. The effect of MVPA was significant in both partition models (stay: $B=-35.31, p$ $=0.02$; switch: $B=-48.24, \quad p=0.004)$. Substituting $30 \mathrm{~min}$ of sedentary behavior, light-intensity activity, or sleep with MVPA yielded faster reaction times on stay trials (sedentary: $B=-29.37, p=0.04$; light: $B=-35.03$, 
Table 1 Sample characteristics

\begin{tabular}{|c|c|c|}
\hline & M & $\pm \mathrm{SD}^{\mathrm{a}}$ \\
\hline & $n$ & $(\%)$ \\
\hline Age (years) & 57.81 & \pm 9.50 \\
\hline Bachelors Degree & 219 & $(80.8)$ \\
\hline Income $\geq \$ 75,000$ per year $(n=256)$ & 193 & $(75.4)$ \\
\hline Employed full-time & 106 & $(39.1)$ \\
\hline Retired & 96 & $(35.4)$ \\
\hline White & 258 & $(95.2)$ \\
\hline Married & 210 & $(77.5)$ \\
\hline \multicolumn{3}{|l|}{ Cancer Stage } \\
\hline 0 & 20 & $(7.4)$ \\
\hline 1 & 110 & $(40.6)$ \\
\hline 2 & 93 & $(34.3)$ \\
\hline 3 & 42 & $(15.5)$ \\
\hline 4 & 6 & $(2.2)$ \\
\hline Months since diagnosis & 95.13 & \pm 73.30 \\
\hline History of chemotherapy only & 42 & $(15.5)$ \\
\hline History of radiation only & 51 & $(18.8)$ \\
\hline History of chemotherapy and radiation & 150 & $(55.4)$ \\
\hline Hormonal therapy (months) & 21.41 & \pm 31.17 \\
\hline Body mass index (BMI) & 26.74 & \pm 5.83 \\
\hline \multicolumn{3}{|l|}{ Accelerometer-derived estimates } \\
\hline Total daily wear time (min) & 1322.80 & \pm 44.09 \\
\hline Wake-time daily wear $(\min )^{b}$ & 912.38 & \pm 61.11 \\
\hline Daily sedentary behavior (min) & 600.03 & \pm 73.24 \\
\hline Daily light-intensity PA (min) & 283.12 & \pm 69.65 \\
\hline Daily moderate-to-vigorous PA (min) & 29.23 & \pm 22.71 \\
\hline Daily sleep duration (min) & 411.16 & \pm 47.39 \\
\hline \multicolumn{3}{|l|}{ Task-Switch } \\
\hline Stay accuracy (\%) & 94.07 & \pm 10.49 \\
\hline Stay reaction time (ms) & 1166.48 & \pm 157.62 \\
\hline Switch accuracy (\%) & 93.46 & \pm 10.60 \\
\hline Switch reaction time (ms) & 1373.29 & \pm 175.64 \\
\hline Trails A time (sec) & 53.96 & \pm 20.04 \\
\hline Trails B time (sec) & 67.67 & \pm 28.55 \\
\hline
\end{tabular}

${ }^{a}$ Mean, Standard Deviation

${ }^{\mathrm{b}}$ Sum of daily time spent in sedentary, light, and MVPA behaviors

$p=0.03$; sleep: $B=-30.47, p=0.055)$ and switch trials (sedentary: $B=-39.49, p=0.02$; light: $B=-41.917, p=$ 0.03; sleep: $B=-39.67, p=0.03$ ). Reallocating sedentary behavior to light-intensity activity or sleep was not associated with faster reaction time on either Task-switch outcome (all $p>0.21$ ).

Trails A total time (Table 4)

In the single effects model, sedentary time was significantly associated with faster Trails A completion $(B=-0.97, p=$
0.03; Table 4), while minutes of light-intensity activity were associated with slower Trails A completion $(B=1.25, p=$ 0.006). MVPA and sleep were not significantly associated with Trails A completion in the single effects model $(p=$ $0.15,0.81)$. However, in the partition model, only MVPA was associated with Trails A completion time $(B=-3.55, p$ $=0.03$ ). Replacing $30 \mathrm{~min}$ of sedentary behavior with 30 min of light-intensity activity was associated with slower Trails A completion $(B=1.55, p=0.002)$. Substituting sedentary behavior with MVPA or sleep was not significantly associated with changes in Trails A time $(p=0.08,0.33)$. When light-intensity activity was replaced with sedentary behavior or MVPA, Trails A times were estimated to be faster (sedentary: $B=-1.55, p=0.002$; MVPA: $B=-4.35$, $p=0.01)$.

\section{Trails B total time (Table 5)}

The single effects of sedentary time, MVPA, light-intensity activity, and sleep were not associated with Trails B completion time (all $p>0.05$; Table 5). In the partition model, MVPA was significantly associated with faster Trails B time $(B=-4.46, p=0.049)$. In the isotemporal substitution model, replacing $30 \mathrm{~min}$ of sedentary time with $30 \mathrm{~min}$ of light-intensity activity or sleep was significantly or marginally associated with slower Trails $B$ completion (light: $B=1.69, p=0.02$; sleep: $B=1.91, p=0.058$ ). Replacing 30 min of sedentary behavior with $30 \mathrm{~min}$ of MVPA did not influence Trails B completion $(p=0.10)$. However, replacing light-intensity activity with MVPA was associated with faster Trails B completion $(B=-5.23, p=0.03)$. A significant association was also observed when MVPA replaced sleep $(B=-5.45$, $p=0.02)$.

\section{Discussion}

The purpose of this study was to investigate the estimated cognitive effects of reallocating daily sedentary behavior to light-intensity physical activity, MVPA, and sleep in BCS. A major strength of this study is the use of objective measures of physical activity, sleep, and cognitive function in a large sample of BCS. Contrary to our hypotheses, findings suggest the benefits of sedentary time replacement to speed of processing and executive function may be restricted to lifestyle behaviors of at least a moderate intensity. Only MVPA was associated with faster task-switch performance in the sedentary time substitution models, while replacing sedentary time with light-intensity activity yielded slower performance on the Trails tasks. Replacing light-intensity activity with MVPA resulted in faster performance on all tasks. These findings are generally consistent with previous studies and may pose important challenges to the design of interventions aimed at mitigating cognitive impairments in BCS. 


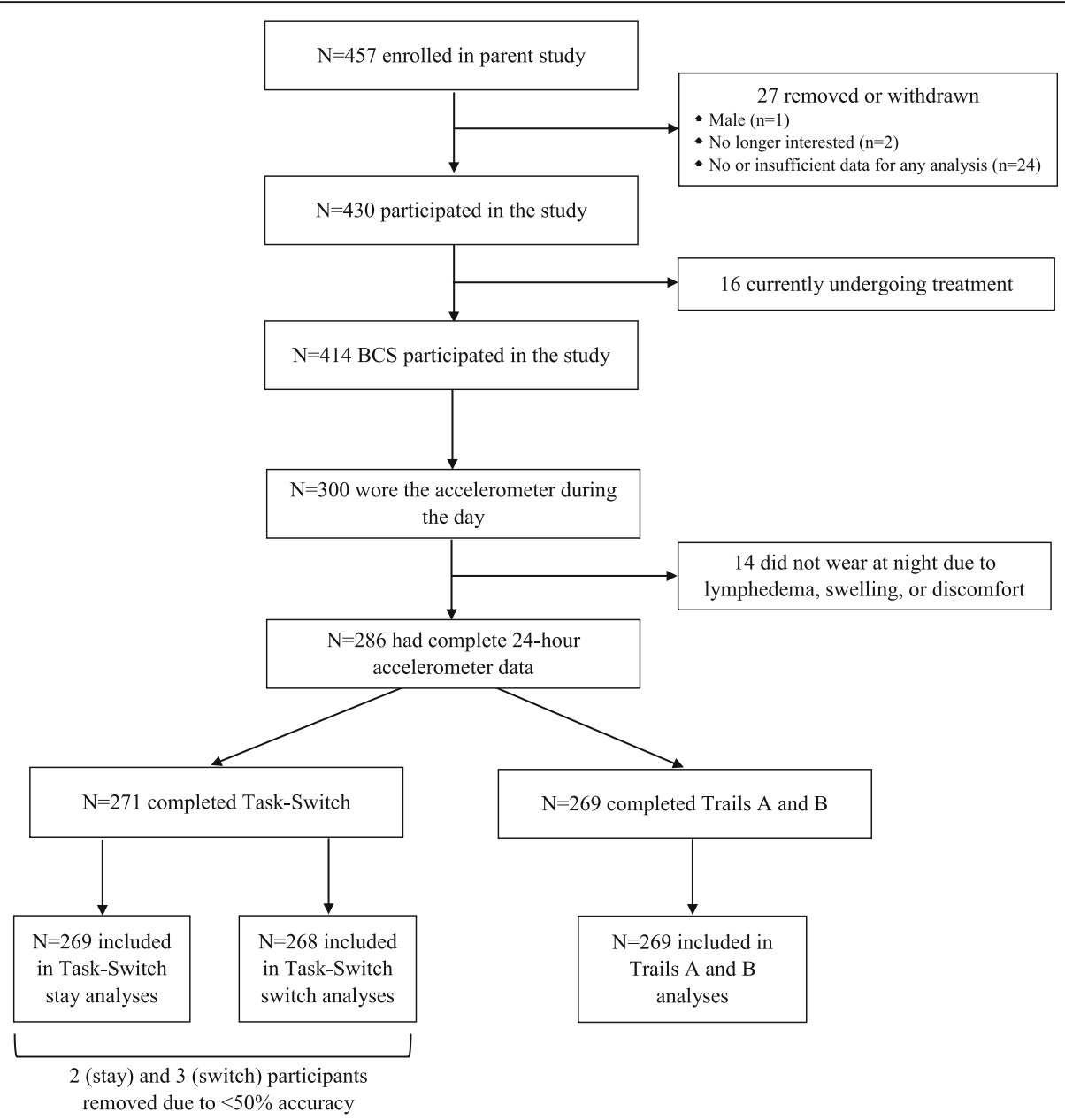

Fig. 1 Flow of Participants through the Study

In one of the few studies also examining the hypothetical effects of sedentary time replacement on cognitive functioning, Fanning and colleagues [22] observed significant improvements in older adults' self-regulatory behaviors and performance on executive function tasks when 30 min of sedentary behavior was substituted with 30 min of MVPA. Further, similar to the present study, replacing sedentary time with light-intensity activity did not lead to improved cognitive performance. The aging literature in general provides strong and consistent evidence in support of moderate-to-vigorous aerobic exercise training for improving cognitive functioning and brain health in older adults [41]. These associations have been replicated in studies of physical activity and CRCI

Table 2 Effects of activity type on task-switch stay reaction times

\begin{tabular}{|c|c|c|c|c|}
\hline \multicolumn{5}{|l|}{ Activity } \\
\hline & Sedentary Time & Light Activity & MVPA & Sleep \\
\hline Single effect & $-1.47[-9.62,6.69]$ & $4.45[-3.79,12.69]$ & $-27.45[-55.85,0.94]$ & $-1.41[-13.50,10.68]$ \\
\hline Partition effect & $-5.94[-18.07,6.18]$ & $-0.28[-12.89,12.33]$ & $-35.31[-64.98,-5.64]$ & $-4.84[-19.46,9.78]$ \\
\hline \multicolumn{5}{|l|}{ Isotemporal effect } \\
\hline Replace sedentary time with... & Dropped & $5.67[-3.36,14.69]$ & $-29.37[-57.96,-0.78]$ & $1.11[-12.07,14.28]$ \\
\hline Replace light activity with... & $-5.67[-14.69,3.36]$ & Dropped & $-35.03[-65.75,-4.32]$ & $-4.56[-17.16,8.04]$ \\
\hline Replace MVPA with... & $29.37[0.78,57.96]$ & $35.03[4.32,65.75]$ & Dropped & $30.47[-0.65,61.59]$ \\
\hline Replace sleep with... & $-1.11[-14.28,12.07]$ & $4.56[-8.04,17.16]$ & $-30.47[-61.59,0.65]$ & Dropped \\
\hline
\end{tabular}

Note. Effects are presented as unstandardized coefficients [95\% confidence interval] of Trails completion in seconds. Single effects and isotemporal models adjusted for age, months of adjuvant hormonal therapy, receipt of chemotherapy, and total time. Bold indicates $p<0.05$ 
Table 3 Effects of activity type on task-switch switch reaction times

\begin{tabular}{|c|c|c|c|c|}
\hline \multicolumn{5}{|l|}{ Activity } \\
\hline & Sedentary Time & Light Activity & MVPA & Sleep \\
\hline Single effect & $2.06[-7.04,11.16]$ & $1.16[-8.06,10.37]$ & $-38.63[-70.18,-7.07]$ & $-0.09[-13.59,13.40]$ \\
\hline Partition effect & $-8.75[-22.26,4.76]$ & $-6.33[-20.38,7.73]$ & $-48.24[-81.30,-15.19]$ & $-8.58[-24.86,7.71]$ \\
\hline \multicolumn{5}{|l|}{ Isotemporal effect } \\
\hline Replace sedentary time with... & Dropped & $2.42[-7.64,12.48]$ & $-39.49[-71.35,-7.64]$ & $0.18[-14.51,14.86]$ \\
\hline Replace light activity with... & $-2.42[-12.48,7.64]$ & Dropped & $-41.91[-76.13,-7.70]$ & $-2.25[-16.29,11.79]$ \\
\hline Replace MVPA with... & $39.49[7.64,71.35]$ & $41.91[7.70,76.13]$ & Dropped & $39.67[4.99,74.34]$ \\
\hline Replace sleep with... & $-0.18[-14.86,14.51]$ & $2.25[-11.79,16.29]$ & $-39.67[-74.34,-4.99]$ & Dropped \\
\hline
\end{tabular}

Note. Effects are presented as unstandardized coefficients [95\% confidence interval] of Trails completion in seconds. Single effects and isotemporal models adjusted for age, months of adjuvant hormonal therapy, receipt of chemotherapy, and total time. Bold indicates $p<0.05$

$[7,8,10,11,42]$. However, none have investigated the cognitive benefits of MVPA in conjunction with, independent of, or in replacement of other activity behaviors in cancer survivors. Our findings fill this knowledge gap with preliminary evidence relative to MVPA, speed of processing, and executive function.

A number of studies have documented beneficial effects of replacing sedentary time with light-intensity activity on cardiometabolic health, body composition, physical function, and psychosocial well-being in older adults and cancer survivors [20, 21, 43-45]. Vallance and colleagues [26], on the other hand, found that only MVPA in 10-min bouts or more were associated with improved health outcomes (i.e., fatigue, HRQoL) in a sample of 149 non-Hodgkin lymphoma survivors. Fatigue is a known correlate of CRCI [27] and has been documented as a potential mediator of the relationship between MVPA and CRCI [10, 46]. Such findings, in combination with those of the present study, may have important implications for the promotion of health-enhancing physical activity in cancer survivors. While sedentary time replacement strategies generally result in increases in light-intensity activity, clinicians may consider approaches that promote MVPA when targeting survivors' cognitive functioning. Certainly the suggestion is not to eliminate whole day approaches to health behavior promotion [47]. However, evidence in support of physical activities of at least a moderate intensity for the improvement of cognitive function is compelling.

Of further interest was the relationship observed between sedentary behavior and light-intensity physical activity in the Trails A and B substitution models. Both Trails A and B completion were estimated to be slower when 30 min of sedentary behavior was replaced with 30 min of light-intensity activity. Despite a plethora of evidence relative to MVPA's influence on cognitive function and brain health across populations, less is known about the influences of sedentary behavior and light-intensity physical activity [17]. Our study is among only a few, to our knowledge, to examine associations between light-intensity physical activity and cognition, and present findings are contrary to those of previous studies. For example, Buchman et al. [48, 49] found that total daily physical activity (actigraphy derived counts) and intensity of physical activity (counts per hour) were associated with greater cognitive function and lower risk of Alzheimer's disease in older adults. While these data suggest that physical activity, regardless of intensity level, has cognitive benefits, the authors did not specifically isolate non-exercise or low-intensity activity. However, more recently, Varma and colleagues [50] linked

Table 4 Effects of activity type on trails A completion

\begin{tabular}{|c|c|c|c|c|}
\hline \multicolumn{5}{|l|}{ Activity } \\
\hline & Sedentary Time & Light Activity & MVPA & Sleep \\
\hline Single effect & $-0.97[-1.85,-0.08]$ & $1.25[0.36,2.14]$ & $-2.29[-5.40,0.83]$ & $-0.16[-1.48,1.16]$ \\
\hline Partition effect & $-0.75[-2.05,0.55]$ & $0.80[-0.55,2.14]$ & $-3.55[-6.76,-0.34]$ & $-0.36[-1.61,1.54]$ \\
\hline \multicolumn{5}{|l|}{ Isotemporal effect } \\
\hline Replace sedentary time with.. & Dropped & $1.55[0.57,2.52]$ & $-2.80[-5.89,0.29]$ & $0.71[-0.72,2.14]$ \\
\hline Replace light activity with... & $-1.55[-2.52,-0.57]$ & Dropped & $-4.35[-7.68,-1.02]$ & $-0.83[-2.19,0.53]$ \\
\hline Replace MVPA with... & $2.80[-0.29,5.89]$ & $4.35[1.02,7.68]$ & Dropped & $3.51[0.13,6.89]$ \\
\hline Replace sleep with... & $-0.71[-2.14,0.72]$ & $0.83[-0.53,2.19]$ & $-3.51[-6.89,-0.13]$ & Dropped \\
\hline
\end{tabular}

Note. Effects are presented as unstandardized coefficients [95\% confidence interval] of Trails completion in seconds. Single effects and isotemporal models adjusted for age, months of adjuvant hormonal therapy, receipt of chemotherapy, and total time. Bold indicates $p<0.05$ 
Table 5 Effects of Activity Type on Trails B Completion

\begin{tabular}{|c|c|c|c|c|}
\hline \multicolumn{5}{|l|}{ Activity } \\
\hline & Sedentary Time & Light Activity & MVPA & Sleep \\
\hline Single effect & $-1.19[-2.41,0.03]$ & $1.02[-0.21,2.26]$ & $-3.16[-7.43,1.12]$ & $0.97[-0.85,2.79]$ \\
\hline Partition effect & $-0.92[-2.71,0.87]$ & $0.77[-1.10,2.63]$ & $-4.46[-8.90,-0.03]$ & $0.99[-1.19,3.17]$ \\
\hline \multicolumn{5}{|l|}{ Isotemporal effect } \\
\hline Replace sedentary time with... & Dropped & $1.69[0.33,3.04]$ & $-3.54[-7.81,0.72]$ & $1.91[-0.07,3.89]$ \\
\hline Replace light activity with... & $-1.69[-3.04,-0.33]$ & Dropped & $-5.23[-9.83,-0.63]$ & $0.22[-1.65,2.10]$ \\
\hline Replace MVPA with... & $3.54[-0.72,7.81]$ & $5.23[0.63,9.83]$ & Dropped & $5.45[0.78,10.12]$ \\
\hline Replace sleep with... & $-1.91[-3.89,0.07]$ & $-0.22[-2.10,1.65]$ & $-5.45[-10.12,-0.78]$ & Dropped \\
\hline
\end{tabular}

Note. Effects are presented as unstandardized coefficients [95\% confidence interval] of Trails completion in seconds. Single effects and isotemporal models adjusted for age, months of adjuvant hormonal therapy, receipt of chemotherapy, and total time. Bold indicates $p<0.05$

low-intensity walking activity, independent of MVPA and self-reported exercise, with hippocampal volume in older adults. It is possible that the effects of light-intensity activity on cognitive processes may be specific to certain domains. The hippocampus is known to control memory processes, while the present study included measures of processing speed and executive function. Further research is warranted to understand how daily behavioral profiles influence cognitive function across domains known to be amenable to physical activity and sleep.

The effects of physical activity on cognitive function are indeed dose-dependent, with MVPA eliciting the greatest behavioral response in cognition $[17,48]$. Our findings in support of MVPA are not unlike those of previous studies focusing on cognitive function [22] or other health outcomes (e.g., fatigue, quality of life) in cancer survivors [26]. However, as these previous studies observed null effects related to light-intensity physical activity, it remains unclear why reallocating sedentary behaviors to light-intensity activity yielded slower performance on both Trails A and B in the present study. It is possible that higher doses of physical activity may have been required to elicit significant cognitive responses in our sample of active and higher functioning BCS. Our eligibility criteria did not exclude physically active BCS, as reflected in the mean MVPA of $30 \mathrm{~min}$ per day. Further, although normative cognitive data for cancer survivors are not currently available, cognitive functioning among participants in the present sample may have been comparable to or even higher than that of the general population of similarly-aged adults [31]. While the present study provides evidence in support of an MVPA prescription for improved cognitive health in BCS, the counterintuitive effects of light-intensity activity warrant further investigation. As MVPA comprises only a small proportion of daily behavior (Table 1), experimental studies specifically testing the effects of light-intensity activities on cognitive function and other health outcomes in BCS may provide the most insightful information [51].

Contrary to our hypothesis, little association between sleep and cognitive performance was observed. Evidence in support of sleep's benefits to cognitive functioning are unequivocal in the general adult population [52, 53]. While no consensus has been reached on the amount of sleep required to optimize cognitive functioning, studies have suggested that habitual sleep durations of fewer than $6 \mathrm{~h}$ or more than 9-9.5 h are related to increased cognitive impairment [54]. In the present study, the vast majority of participants had sleep durations of $6-9 \mathrm{~h}$ per night $(82.0 \%)$, indicating generally sufficient sleep across the sample. While sleep disturbances are thought to be more prevalent in BCS when compared with non-cancer populations, Budhrani and colleagues [55] demonstrated in a recent review that total sleep time may not differ between BCS and non-cancer adults. Additionally, other sleep metrics may better explain the influence of sleep on cognitive function across ages and populations [19]. In future studies exploring the effects of sleep on CRCI, investigations of other sleep quality outcomes, such as wakefulness after sleep onset, sleep onset latency, and daytime dysfunction, may be more informative than sleep duration alone.

\section{Limitations}

This study had a number of strengths, including enrollment of a national sample of BCS, objective measures of daytime and sleep behaviors via actigraphy, and objective measures of cognitive functioning. Despite these strengths, this study also had limitations. First, participants represented a homogeneous population of Caucasian, well-educated, and affluent breast cancer survivors. Therefore, generalizability of the results to other populations of breast cancer survivors is limited. Additionally, several participants reported daytime naps on their accelerometer log. We did not ask participants to record such information and, therefore, did not remove any reported daytime nap periods from activity calculations. As such, bouts of daytime sleep were most likely 
categorized as sedentary time or non-wear. The effects of sleep on health are generally distinct from sedentary behavior [21]. Further, napping has been associated with improved cognitive function; yet, prolonged napping may also be an indicator of underlying health conditions [56]. Therefore, we not only were unable to test the effects of daytime sleep on cognitive function, but napping, if widespread across the sample, may also have inhibited our ability to full test the effects of sedentary behavior on cognitive function. Because sleep dysfunction is common among cancer survivors [55], efforts to understand health conditions associated with daytime sleep in cancer survivors and the effects of napping on health outcomes, such as cognitive function, are needed.

Similarly, our objective measure did not provide us with any contextual information about sedentary behaviors. Television viewing, for example, has consistently been associated with poorer health outcomes in older adults, while social and cognitive sitting activities, such as talking with friends, reading, or completing a puzzle, may have neuroprotective health effects [57-59]. Further research dissecting daily sedentary behavior among BCS may help us to better explain the effects of light-intensity activity observed in the present study. Finally, causal associations among variables cannot be discerned in the present study due to the cross-sectional design and hypothetical modeling of sedentary time replacement. Prospective and experimental studies are needed to further test interactions among behaviors across the 24-h period.

\section{Conclusions}

Isotemporal substitution models in which sedentary behavior was replaced with physical activity yielded improvements in BCS's performance on cognitive tasks measuring speed of processing and executive function. More importantly, these improvements were dose-dependent, with MVPA conferring the most benefit and light-intensity activity resulting in declined performance in some models. In fact, reallocating MVPA to sedentary activity, light-intensity activity, or sleep was consistently associated with poorer cognitive performance. Further testing of interactions among sedentary behavior, light-intensity physical activity, MVPA, and sleep on CRCI is needed to inform the design of cancer rehabilitation strategies targeting cognitive function in cancer survivors.

\section{Additional file}

Additional file 1: Supplementary File (Additional Questionnaires). (PDF $78 \mathrm{~kb})$

\section{Abbreviations}

BCS: Breast cancer survivors; CRCl: Cancer-related cognitive impairment; HRQoL: Health-related quality of life; MVPA: Moderate-to-vigorous physical activity; US: United States

\section{Acknowledgements}

We would like to thank Allen Best and the rest of the team at Digital Artefacts for their work on this project.

\section{Funding}

Dr. Ehlers and this project were supported by an American Cancer Society (ACS) Postdoctoral Fellowship (PF-16-021-01-CPPB) at the University of Illinois at Urbana-Champaign. ACS was not involved in the design of the study; collection, analysis, and interpretation of data; or writing of the manuscript.

\section{Availability of data and materials}

The datasets used and/or analyzed during the current study are available from the corresponding author on reasonable request.

\section{Authors' contributions}

DKE conceived the present study; collected, processed, and analyzed the data; and wrote the manuscript. JF contributed to the conception of the present study, data analysis, and manuscript writing. ES assisted with data analysis and interpretation and with manuscript writing. SA and JC assisted with data collection, processing, and interpretation. JS developed the platform through which the data were collected. AFK is the secondary senior author on this study and provided mentorship relative to the overall study design, data analysis, and manuscript development. EM is the senior author who, with the first author, conceived the overall study and provided supervision and mentorship relative to data analysis and manuscript development. All authors have read and approved the final manuscript.

\section{Ethics approval and consent to participate}

This study was approved by the Institutional Review Board at the University of Illinois at Urbana-Champaign. All participants digitally signed the iPad-based consent form prior to enrollment in the study.

\section{Consent for publication}

This section is not applicable to the present study.

\section{Competing interests}

The authors declare that they have no competing interests.

\section{Publisher's Note}

Springer Nature remains neutral with regard to jurisdictional claims in published maps and institutional affiliations.

\section{Author details}

${ }^{1}$ University of South Carolina, Columbia, SC 29208, USA. ${ }^{2}$ Wake Forest School of Medicine, Winston-Salem, NC, USA. ${ }^{3}$ National Cancer Institute, Rockville, MD, USA. ${ }^{4}$ University of Illinois at Urbana-Champaign, Urbana, IL, USA. ${ }^{5}$ Pfizer Incorporated, Cambridge, MA, USA. ${ }^{6}$ Digital Artefacts, lowa City, IA, USA.

${ }^{7}$ Northeastern University, Boston, MA, USA.

Received: 12 December 2017 Accepted: 18 June 2018

Published online: 25 June 2018

\section{References}

1. DeSantis CE, Lin CC, Mariotto AB, Siegel RL, Stein KD, Kramer JL, et al. Cancer treatment and survivorship statistics, 2014. CA Cancer J Clin. 2014;64: 252-71.

2. Myers JS. Chemotherapy-related cognitive impairment: the breast cancer experience. Oncol Nurs Forum. 2011:39:E31-40.

3. Ahles TA, Root JC, Ryan EL. Cancer- and cancer treatment-associated cognitive change: an update on the state of the science. J Clin Oncol. 2012; 30:3675-86.

4. Magnuson A, Allore H, Cohen HJ, Mohile SG, Williams GR, Chapman A, et al. Geriatric assessment with management in cancer care: current evidence and potential mechanisms for future research. J Geriatr Oncol. 2016;7:242-8.

5. Ehlers $D$, Trinh L, McAuley E. The intersection of cancer and aging: implications for physical activity and cardiorespiratory fitness effects on cognition. Expert Rev Qual Life Cancer Care. 2016;1:347-50.

6. Von AD, Jansen C, Allen DH, Schiavone RM, Wulff J. Putting evidence into practice: evidence-based interventions for cancer and cancer treatmentrelated cognitive impairment. Clin J Oncol Nurs. 2011;15:607-15. 
7. Zimmer P, Baumann FT, Oberste M, Wright P, Garthe A, Schenk A, et al. Effects of exercise interventions and physical activity behavior on cancer related cognitive impairments: a systematic review. Biomed Res Int. 2016; 2016:1820954.

8. Hartman SJ, Nelson SH, Myers E, Natarajan L, Sears DD, Palmer BW, et al. Randomized controlled trial of increasing physical activity on objectively measured and self-reported cognitive functioning among breast cancer survivors: the memory \& motion study. Cancer. 2018;24(1):192-202.

9. Myers JS, Erickson Kl, Sereika SM, Bender CM. Exercise as an intervention to mitigate decreased cognitive function from cancer and cancer treatment: an integrative review. Cancer Nurs. 2017. Epub ahead of print

10. Ehlers DK, Aguiñaga S, Cosman J, Severson J, Kramer AF, McAuley E. The effects of physical activity and fatigue on cognitive performance in breast cancer survivors. Breast Cancer Res Treat. 2017;165:699-707.

11. Marinac CR, Godbole S, Kerr J, Natarajan L, Patterson RE, Hartman SJ. Objectively measured physical activity and cognitive functioning in breast cancer survivors. J Cancer Surviv. 2015;9:230-8.

12. Lynch BM, Dunstan DW, Healy GN, Winkler E, Eakin E, Owen N. Objectively measured physical activity and sedentary time of breast cancer survivors, and associations with adiposity: findings from NHANES (2003-2006). Cancer Causes Control. 2010:21:283-8.

13. Lynch BM, Dunstan DW, Vallance JK, Owen N. Don't take cancer sitting down: a new survivorship research agenda. Cancer. 2013;119:1928-35.

14. Thraen-Borowski KM, Gennuso KP, Cadmus-Bertram L. Accelerometerderived physical activity and sedentary time by cancer type in the United States. PLoS One. 2017;12:e0182554.

15. Healy GN, Wijndaele K, Dunstan DW, Shaw JE, Salmon J, Zimmet PZ, et al. Objectively measured sedentary time, physical activity, and metabolic risk: the Australian diabetes, obesity and lifestyle study (AusDiab). Diabetes Care. 2008:31:369-71.

16. Owen N, Sparling PB, Healy GN, Dunstan DW, Matthews CE. Sedentary behavior: emerging evidence for a new health risk. Mayo Clin Proc. 2010;85:1138-41.

17. Voss MW, Carr LJ, Clark R, Weng T. Revenge of the "sit" II: does lifestyle impact neuronal and cognitive health through distinct mechanisms associated with sedentary behavior and physical activity? Ment Health Phys Act. 2014;7:9-24.

18. U.S. Department of Health and Human Services. 2008 Physical activity guidelines for Americans. Pres Counc Phys Fit Sport Res Dig. 2008;9:1-8.

19. Scullin MK, Bliwise DL. Sleep, cognition, and normal aging. Perspect Psychol Sci. 2015;10:97-137.

20. Buman MP, Hekler EB, Haskell WL, Pruitt L, Conway TL, Cain KL, et al. Objective light-intensity physical activity associations with rated health in older adults. Am J Epidemiol. 2010;172:1155-65.

21. Buman MP, Winkler EAH, Kurka JM, Hekler EB, Baldwin CM, Owen N, et al. Reallocating time to sleep, sedentary behaviors, or active behaviors: associations with cardiovascular disease risk biomarkers, NHANES 2005-2006. Am J Epidemiol. 2014;179:323-34

22. Fanning J, Porter G, Awick EA, Ehlers DK, Roberts SA, Cooke G, et al. Replacing sedentary time with sleep, light, or moderate-to-vigorous physical activity: effects on self-regulation and executive functioning. J Behav Med. 2017;40:332-42.

23. Phillips SM, Awick EA, Conroy DE, Pellegrini CA, Mailey EL, McAuley E. Objectively measured physical activity and sedentary behavior and quality of life indicators in survivors of breast cancer. Cancer. 2015:121:4044-52.

24. Van Roekel EH, Winkler EAH, Bours MJL, Lynch BM, Willems PJB, Meijer K, et al. Associations of sedentary time and patterns of sedentary time accumulation with health-related quality of life in colorectal cancer survivors. Prev Med Reports. 2016;4:262-9.

25. Trinh L, Amireault S, Lacombe J, Sabiston CM. Physical and psychological health among breast cancer survivors: interactions with sedentary behavior and physical activity. Psycho Oncology. 2015;24:1279-85.

26. Vallance JK, Buman MP, Lynch BM, Boyle T. Reallocating time to sleep, sedentary, and active behaviours in non-Hodgkin lymphoma survivors: associations with patient-reported outcomes. Ann Hematol. 2017;96:749-55.

27. Janelsins MC, Kohli S, Mohile SG, Usuki K, Ahles TA, Morrow GR. An update on cancer- and chemotherapy-related cognitive dysfunction: current status. Semin Oncol. 2011:38:431-8.

28. Hardcastle SJ, Cohen PA. Effective physical activity promotion to survivors of cancer is likely to be home based and to require oncologist participation. J Clin Oncol. 2017. https://doi.org/10.1200/JCO.2017.74.6032.
29. Larkey LK, Roe DJ, Weihs KL, Jahnke R, Lopez AM, Rogers CE, et al. Randomized controlled trial of qigong/tai chi easy on cancer- related fatigue in breast cancer survivors. Ann Behav Med. 2015;49:165-76.

30. Velthuis MJ, Agasi-Idenburg SC, Aufdemkampe G, Wittink HM. The effect of physical exercise on cancer-related fatigue during cancer treatment: a metaanalysis of randomised controlled trials. Clin Oncol. 2010;22:208-21.

31. Lee H, Baniqued PL, Cosman J, Mullen S, McAuley E, Severson J, et al. Examining cognitive function across the lifespan using a mobile application. Comput Human Behav. 2012;28:1934-46.

32. Troiano RP, Berrigan D, Dodd KW, Mâsse LC, Tilert T, McDowell M. Physical activity in the United States measured by accelerometer. Med Sci Sports Exerc. 2008;40:181-8.

33. Freedson P, Melanson E, Sirard J. Calibration of the computer science and applications, Inc. accelerometer. Med Sci Sports Exerc. 1998;30:777-81.

34. Sadeh A, Sharkey KM, Carskadon MA. Activity-based sleep-wake identification: an empirical test of methodological issues. Sleep. 1994;3:201-7

35. Mekary RA, Willett WC, Hu FB, Ding EL. Isotemporal substitution paradigm for physical activity epidemiology and weight change. Am J Epidemiol. 2009;170:519-27.

36. Mekary RA, Lucas M, Pan A, Okereke OI, Willett WC, Hu FB, et al. Isotemporal substitution analysis for physical activity, television watching, and risk of depression. Am J Epidemiol. 2013;178:474-83.

37. Clark R, Hazeltine E, Freedberg M, Voss MW. Age differences in episodic associative learning. Psychol Aging. 2018:33:144-57.

38. Monsell S. Task switching. Trends Cogn Sci. 2003;7:134-40.

39. Battery AIT. Manual of directions and scoring. Washington, D.C.: War Department, Adjutant General's Office; 1994.

40. Janelsins MC, Kesler SR, Ahles TA, Morrow GR. Prevalence, mechanisms, and management of cancer-related cognitive impairment. Int Rev Psychiatry. 2014;26:102-13

41. Northey JM, Cherbuin N, Pumpa KL, Smee DJ, Rattray B. Exercise interventions for cognitive function in adults older than 50: a systematic review with meta-analysis. 2018:52(3):154-60.

42. Cooke GE, Wetter NC, Banducci SE, Mackenzie MJ, Zuniga KE, Awick EA, et al. Moderate physical activity mediates the association between white matter lesion volume and memory recall in breast cancer survivors. PLoS One. 2016:11:e0149552.

43. Blair CK, Morey MC, Desmond RA, Cohen HJ, Sloane R, Snyder DC, et al. Light-intensity activity attenuates functional decline in older cancer survivors. Med Sci Sports Exerc. 2014;46:1375-83. NIH Public Access

44. Boyle T, Vallance JK, Buman MP, Lynch BM. Reallocating time to sleep, sedentary time, or physical activity: associations with waist circumference and body mass index in breast cancer survivors. Cancer Epidemiol Biomarkers Prev. 2017:26:254-60

45. Sylvester BD, Ahmed R, Amireault S, Sabiston CM. Changes in lightmoderate-, and vigorous-intensity physical activity and changes in depressive symptoms in breast cancer survivors: a prospective observational study. Support Care Cancer. 2017:25:3305-12.

46. Phillips SM, Lloyd GR, Awick EA, McAuley E. Relationship between selfreported and objectively measured physical activity and subjective memory impairment in breast cancer survivors: role of self-efficacy, fatigue and distress. Psychooncology. 2017:26:1390-9.

47. Dunstan DW, Owen N. New exercise prescription: don't just sit there: stand up and move more, more often. Arch Intern Med. 2012;172:500.

48. Buchman AS, Boyle PA, Yu L, Shah RC, Wilson RS, Bennett DA. Total daily physical activity and the risk of $A D$ and cognitive decline in older adults. Neurol Int. 2012;78:1323-9.

49. Buchman AS, Wilson RS, Bennett DA. Total daily activity is associated with cognition in older persons. Am J Geriatr Psychiatry. 2008;16:697-701.

50. Varma VR, Chuang Y-F, Harris GC, Tan EJ, Carlson MC. Low-intensity daily walking activity is associated with hippocampal volume in older adults. Hippocampus. 2015;25:605-15.

51. Phillips SM, Dodd KW, Steeves J, McClain J, Alfano CM, McAuley E. Physical activity and sedentary behavior in breast cancer survivors: new insight into activity patterns and potential intervention targets. Gynecol Oncol. 2015; 138:398-404.

52. Schmutte T, Harris S, Levin R, Zweig R, Katz M, Lipton R. The relation between cognitive functioning and self-reported sleep complaints in nondemented older adults: results from the bronx aging study. Behav Sleep Med. 2007:5:39-56. 
53. Kronholm E, Sallinen M, Suutama T, Sulkava R, Era P, Partonen T. Selfreported sleep duration and cognitive functioning in the general population. J Sleep Res. 2009;18:436-46.

54. Ohayon MM, Vecchierini M-F. Normative sleep data, cognitive function and daily living activities in older adults in the community. Sleep. 2005;28:981-9.

55. Budhrani PH, Lengacher CA, Kip K, Tofthagen C, Jim H. An integrative review of subjective and objective measures of sleep disturbances in breast cancer survivors. Clin J Oncol Nurs. 2015;19:185-92.

56. Ficca G, Axelsson J, Mollicone DJ, Muto V, Vitiello MV. Naps, cognition and performance. Sleep Med Rev. 2010;14(4):249-58.

57. Hamer M, Stamatakis E. Prospective study of sedentary behavior, risk of depression, and cognitive impairment. Med Sci Sports Exerc. 2014;46:718-23.

58. Falck RS, Davis JC, Liu-Ambrose T. What is the association between sedentary behaviour and cognitive function? A systematic review. Br J Sports Med. 2017:51:800-11.

59. Katzmarzyk PT. Physical activity, sedentary behavior, and health: paradigm paralysis or paradigm shift? Diabetes. 2010;59:2717-25.

Ready to submit your research? Choose BMC and benefit from:

- fast, convenient online submission

- thorough peer review by experienced researchers in your field

- rapid publication on acceptance

- support for research data, including large and complex data types

- gold Open Access which fosters wider collaboration and increased citations

- maximum visibility for your research: over $100 \mathrm{M}$ website views per year 